\title{
Selective Recording of Urinary Bladder Fullness from the Extradural Sacral Roots*
}

\author{
B. Metcalfe, N. Granger, J. Prager, S. Sadrafshari, T. Grego, J. Taylor and N. Donaldson
}

\begin{abstract}
Managing the urinary bladder is of primary importance to clinicians and patients after trauma to the spinal cord. Sacral Anterior Root Stimulators that control the bladder have been available as clinical technology for many years, however these devices cannot measure the fullness of the urinary bladder or detect the onset of reflex voiding. In order to address this fundamental limitation, it is necessary to develop a method for recording the neural signals that encode bladder fullness. This paper presents a proof of concept technique for recording bladder afferents from the extradural sacral roots using a multiple electrode cuff. Results are provided from acute in-vivo experiments performed in sheep.
\end{abstract}

\section{INTRODUCTION}

Spinal Cord Injury (SCI) affects over 12,000 new patients a year in the USA and about 1,000 in the UK [1]. This amounts to a total of approximately $£ 1$ billion on the NHS budget annually [see www.spinal-research.org]. Worldwide, Spinal Cord Injury (SCI) affects about 2.5 million people with approximately 130,000 new cases each year.

Managing the urinary bladder is of first importance after trauma to the spinal cord, since kidney damage due to high bladder pressures and/or infection was historically a usual cause of death following such an injury [2][3]. At present the most common method for bladder management after SCI is intermittent catheterisation; this is expensive, invasive, and infection prone. Clinical presentation of the infection may be graded, from mild dysuria and pain (grade 1) to urosepsis and death (grade 8) [4].

As an alternative to catheterisation, a neuroprosthesis for controlling the bladder was developed by Giles Brindley over 30 years ago [5]. This is currently manufactured by Finetech Medical Ltd and approximately 4,000 devices have been implanted worldwide. This device employs Sacral Anterior Root Stimulation (SARS) to control the urinary bladder, but has the downside of requiring rhizotomy of the sacral posterior roots to prevent reflex incontinence. This is unpopular because it inflicts further trauma to the nerves and removes remaining sexual function.

The need for a rhizotomy arises because as the bladder fills it undergoes reflex contractions that are normally inhibited by the brain. With SCI these contractions are not blocked, and spontaneous voiding occurs. An improved neuroprosthesis is required that can sense and stop this voiding, removing the need for a rhizotomy. Such a device would require recording and decoding of the electrical signals within the sacral roots

*Research supported by the Engineering and Physical Sciences Research Council (EPSRC), UK via EP/P018947/1.

B. Metcalfe, S. Sadrafshari and J. Taylor are with the Department of Electronic and Electrical Engineering, University of Bath, Bath BA2, UK. that encode bladder pressure and fullness, which does not currently exist.

Existing research has shown that it is possible to record neural signals that encode bladder fullness from both the pelvic nerve and the sacral roots using an implantable cuff electrode [6]. Signals recorded from the pelvic nerve have a greater amplitude than those recorded from the sacral roots, although these amplitudes are still very low. However, accessing the pelvic nerve is difficult and may not be straightforward in humans. The sacral roots meanwhile are easily accessible via a laminectomy of the sacral vertebra, the same procedure is how the Brindley Device is currently implanted in humans.

This paper presents preliminary results from acute in-vivo experiments performed in sheep, wherein a Multiple Electrode Cuff(MEC) was implanted on the extra-dural sacral roots and Velocity Selective Recording (VSR) was used to selectively enhance bladder afferents.

\section{METHODS}

\section{A. Surgical Approach}

All the experimental procedures detailed in this paper were performed in accordance with the United Kingdom Animals (Scientific Procedures) Act 1986 following approval from the relevant institutional ethics committees.

An acute experiment was performed on an adult female sheep (weight $45 \mathrm{~kg}$ ). The ewe underwent general anaesthesia involving: a pre-operative transdermal Fentanyl patch, premedication with Ketamine $(7.5 \mathrm{mg} / \mathrm{kg})$ and Midazolam $(0.5$ $\mathrm{mg} / \mathrm{kg}$ ), induction with Propofol, and maintenance on Sevoflurane in oxygen. Intraoperative Fentanyl \& Ketamine analgesia were provided as required. A lumbosacral dorsal laminectomy was performed to expose the sacral roots and each root was identified based on anatomical landmarks (e.g. lumbosacral junction) and by their responses to electrical stimulation. At the end of the experiment the animal was terminated by overdose of pentobarbital.

\section{B. Cystometry}

Bladder filling and pressure recording was achieved via the use of a commercial cystometry system (Medica S.p.A PicoSmart). The bladder was catheterised with a 12Fr Foley catheter and the balloon inflated to prevent leaking of fluid from the bladder. Bladder volume and pressure were recorded continuously via a proprietary urinary catheter.

N. Donaldson and T. Grego are with the Department of Medical Physics and Bioengineering, University College London, London WC1, UK.

N. Granger and J. Prager are with The Royal Veterinary College, Hawkshead Lane, Brookmans Park, Hatfield AL9, UK. 


\section{Electrodes}

A custom recording MEC was fabricated with ten contacts formed from stainless steel rings (spacing $1.5 \mathrm{~mm}$ ) embedded in silicone with adjustable diameters $\varnothing=1-2.4 \mathrm{~mm}$ (Fig. 1). The cuff design is tightly constrained by the space available around the extradural roots, the number of electrodes, and the electrode spacing. The electrical connection to the cuff was established via implantable Cooper cables terminated with Lemo plugs. Stimulation electrodes were bipolar extradural surgical probes (Finetech Medical BSC259). Each root was stimulated by holding the back of the hooks against the root or by carefully lifting the root in the hooks.

\section{Stimulation \& Recording}

Electrical stimulation of the roots was performed before implanting the MEC at $3 \mathrm{~Hz}$ from $1-20 \mathrm{~V}$ using an adjustable and battery powered surgical stimulator (Finetech Medical Model BSD260). Each root was stimulated in turn with increasing amplitudes and once the root providing the largest increase in bladder pressure was identified the MEC was implanted onto that root.

The implanted cuff was connected to a bank of custom-made low noise pre-amplifiers and then to a second stage of commercial amplifiers (Warner Instruments DP-304A). The pre-amplifiers were fabricated using the Austria Microsystems $0.35 \mu \mathrm{m}$ process and employ a 2-stage, ac coupled architecture using MOS transistors in the front end stage, providing high input impedance. An $R C$ interface filter was placed between the amplifiers and the MEC to suppress interfering signals. The overall amplifier gain was $92 \mathrm{~dB}$ and the system bandwidth was $100 \mathrm{~Hz}-50 \mathrm{kHz}$. The input referred noise floor with the inputs shorted was approximately $1.3 \mu \mathrm{V}$ RMS per channel. The expected amplitudes of the action potentials recorded with a cuff are very low (typically $1-10 \mu \mathrm{V}$ for single action potentials and $10-100 \mu \mathrm{V}$ for compound action potentials).

The amplified and filtered signals were digitised using a bank of high-speed analogue to digital converters (NI9222 mounted in cDAQ-9178 by National Instruments, Austin, TX, USA) providing simultaneous sampling of all channels at a

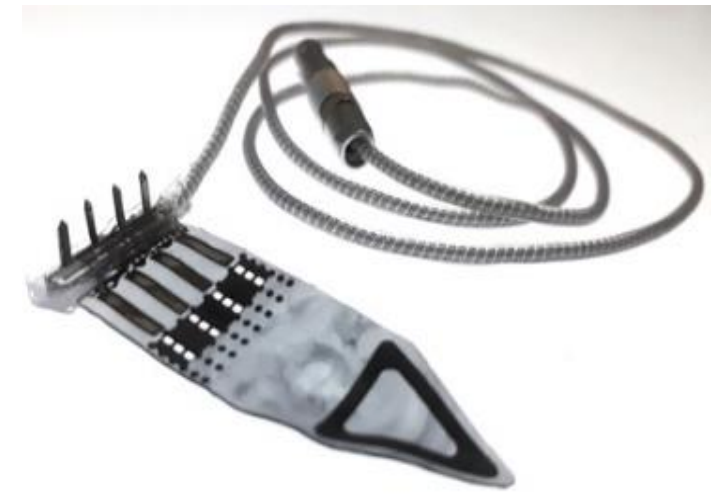

Figure 1: An example of the type of MEC used in this experiment. The example shown is a four-electrode type fitted with a Cooper cable and Lemo connector. sample rate of $100 \mathrm{kS} / \mathrm{s}$ with 16-bit resolution. The high sampling rate (approximately 10 times the Nyquist frequency for the accepted signal bandwidth of $10 \mathrm{kHz}$ ) was necessary due to the small conduction delays of the faster action potentials. The electrodes were connected in a bipolar fashion (see Fig. 2) and the amplifier reference was connected to the epidermis via the surgical retractors.

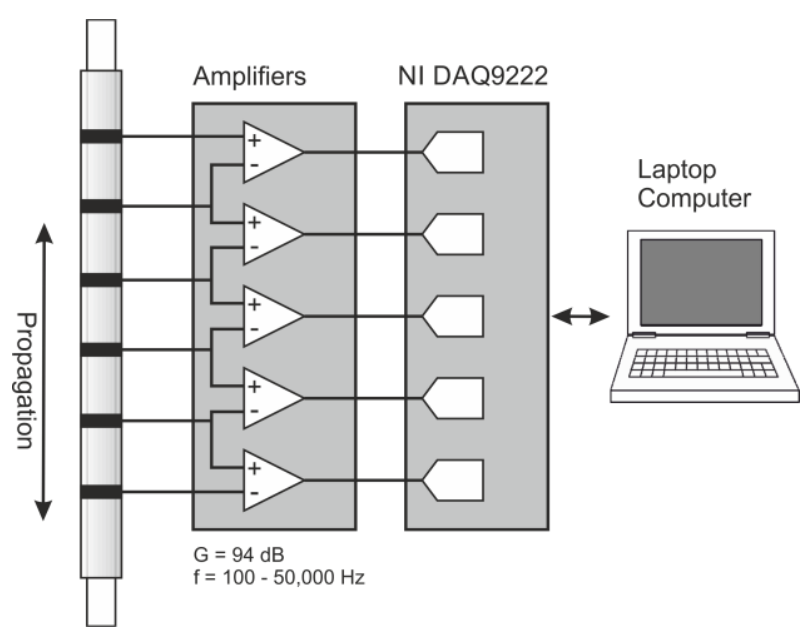

Figure 2: Simplified recording configuration (reduced electrode count) illustrating the bipolar amplifier arrangement.

\section{E. Data Analysis}

After digitisation, all recordings were stored in data files for later analysis. Initial artefact rejection was performed by comparing each signal with the long term mean and standard deviation on a sample per sample basis. If a sample value exceeded a pre-set threshold value (mean \pm 3.5 the standard deviation) that sample was replaced with one equal to the long-term mean.

After artefact removal the signals from each dipole were selectively combined using a process known as Delay-and$A d d$. Each bipolar recording $V_{B i}$ was delayed relative to the end recording by an interval that depends on both the electrode spacing and the action potential conduction velocity of interest. So, if the delay inserted between the first two channels $\left(V_{B 1}, V_{B 2}\right)$ is $d t$ then the delay inserted between the first and third channels $\left(V_{B 1}, V_{B 3}\right)$ is $2 d t$ and so on. After insertion of the artificial delay, which can be negative, the signals are summed together. Clearly, the result of this summation will be largest if the artificial delay cancels out the naturally occurring delay caused by the finite conduction velocity of the action potential.

There are many advantages of this process. Firstly, by using both positive and negative values for $d t$ it is possible to isolate afferent and efferent neural activity. Secondly, when delayand-add is used, and the noise sources are uncorrelated (a reasonable assumption), then there is an increase in effective signal to noise ratio of approximately $\sqrt{N}$ where $N$ is the number of signals [7]. Finally, any contaminating signals that appear as common mode are automatically rejected. 

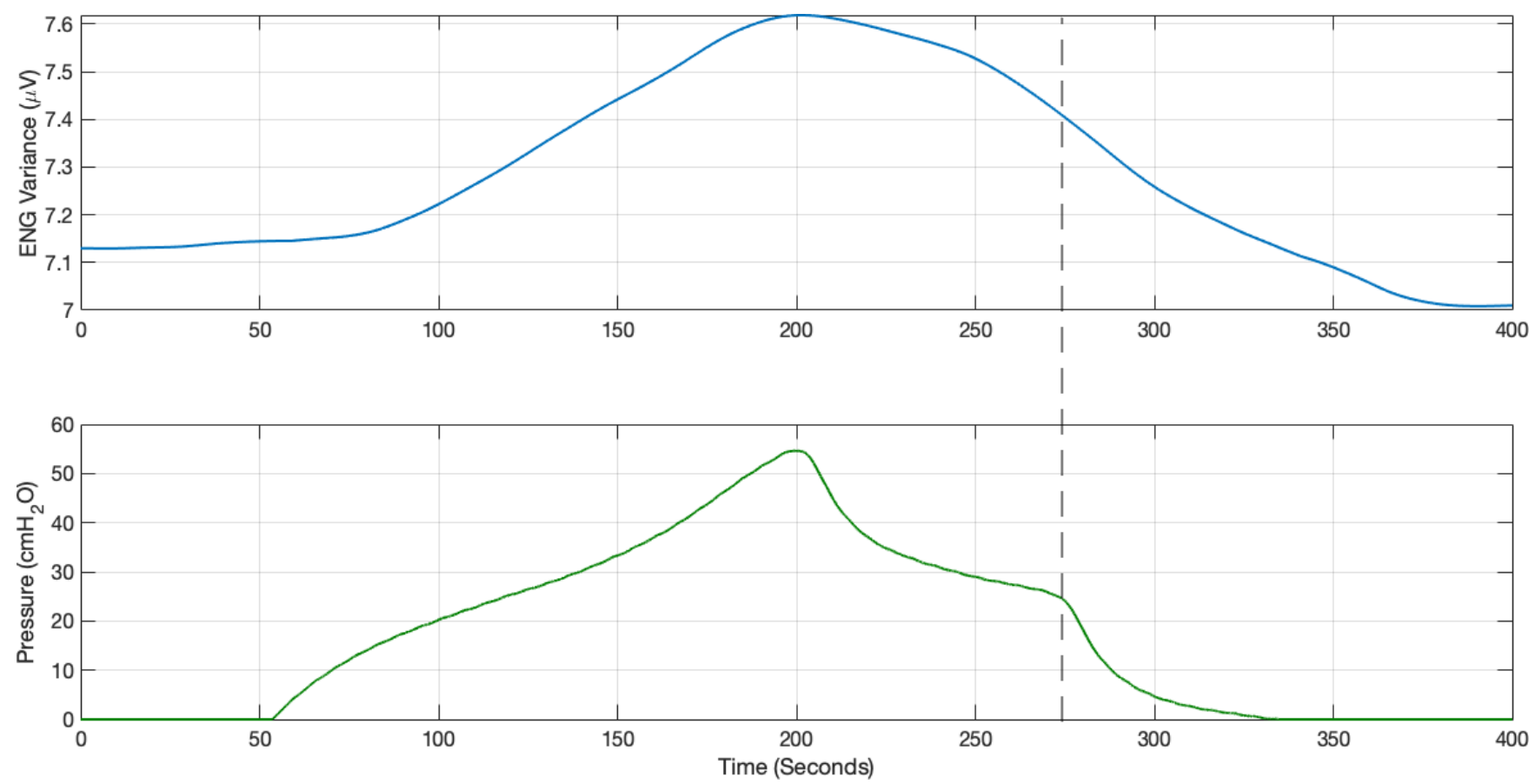

Figure 3: Change in ENG variance during bladder filling. The bladder pressure follows three distinct phases, filling, resting, and voiding. The dashed vertical marker indicates opening of the catheter to void the bladder. There is a clear correlation between both the bladder pressure and the ENG variance.

Once the bipolar signals had been summed together the variance of the resulting signal was found by use of a moving variance operator using a $200 \mathrm{~ms}$ sliding window with a single sample overlap. Finally, the variance signal was smoothed by applying a moving average (mean) function. The resulting signal was then directly compared to the recorded bladder pressure.

\section{F. Experimental Protocol}

The MEC recording cuff was implanted on the root that gave the strongest bladder response to electrical stimulation, and then both 2 -wire and 4-wire impedance measurements were taken to ascertain the electrode contact impedance and the volume conductor impedance. Initial validation of the recording interface was performed by connecting a loudspeaker to one of the amplifier outputs and listing for audible changes while manually stimulating the dermatomes. This dermatome test was repeated before, during, and after each experiment.

The bladder was infused with room temperature Hartmann's solution at a rate of $100 \mathrm{~mL} / \mathrm{min}$ to a maximum pressure of $60 \mathrm{cmH}_{2} \mathrm{O}$. The bladder was then left for a short period of time before being emptied in an uncontrolled manner by opening the catheter. The bladder pressure, the rectal pressure, and the ENG from the MEC were recorded continuously throughout each bladder filling. In one experiment the sacral root was cut proximal to the bladder in order to validate that the recorded ENG signals were afferent.

\section{RESULTS}

\section{A. Urodynamic Function}

Multiple pre-operative cystometries confirmed the ability to obtain bladder pressures of at least $60 \mathrm{cmH}_{2} \mathrm{O}$ by infusing, on average, $260 \mathrm{~mL}$ of fluid into the bladder. In all cases once the pump had been stopped the bladder accommodated and the pressure fell to a stable level that was significantly below the peak pressure. The bladder was left infused during the surgical approach with no apparent loss of bladder muscle tone or pressure throughout.

\section{B. Electrode Impedances \& Stimulation}

Electrode impedances were measured at $1 \mathrm{kHz}$ using a $100 \mathrm{mV}$ compliance voltage. Two-wire measurements showed impedances of approximately $2.5 \mathrm{k} \Omega$ at -45 degrees with consistency over all electrodes. The four-wire Kelvin measurement (electrodes 1-2-9-10) showed a very consistent impedance of approximately $598 \Omega$ at -3 degrees. The impedances were stable throughout the duration of the experiments.

The roots that gave the clearest increase in bladder pressure with electrical stimulation were S3 Left and Right, with an average increase in pressure of $15 \mathrm{cmH}_{2} \mathrm{O}$ for each root and $25 \mathrm{cmH}_{2} \mathrm{O}$ when both roots were stimulated bilaterally. Stimulation of S2 and S4 gave weaker pressure increases of around $5 \mathrm{cmH}_{2} \mathrm{O}$, whereas $\mathrm{S} 1$ gave no increase in bladder pressure but did cause large muscle contractions via the sciatic nerve. Thus, the MEC was implanted onto the S3 Right root. 


\section{Nerve Recordings}

The nerve recordings were processed using the methods described in Section II, assuming a conduction velocity for the bladder afferents of around $40 \mathrm{~m} / \mathrm{s}$ (and thus a conduction delay from one electrode to another of $d t=37.5 \mu \mathrm{s}$ ) [8]. The variance of the ENG within the $S 3$ root remained steady at a baseline value of approximately $7 \mu \mathrm{V}$ before bladder filling commenced. During bladder filling both the ENG variance and the pressure rose in tandem to a peak pressure of $55 \mathrm{cmH}_{2} \mathrm{O}$ and a peak variance of $7.8 \mu \mathrm{V}$, corresponding to an $11 \%$ increase in the ENG variance from an empty to full bladder (Fig. 3). The ENG variance increase on a single dipole without using the delay-and-add process was less than $3 \%$. Once the peak pressure had been reached the cystometry pump was stopped $(\mathrm{T}=200)$ and the bladder was allowed to accommodate, during which time the pressure slowly fell before stabilising at around $30 \mathrm{cmH}_{2} \mathrm{O}$. Finally, the catheter was opened to void the bladder $(\mathrm{T}=270$, dashed vertical marker), at which point the ENG variance decreased to a level that was just below the starting baseline value. After cutting the root proximal to the bladder the ENG variance stayed constant during subsequent bladder fillings, indicating that the change in variance is likely the results of bladder afferents.

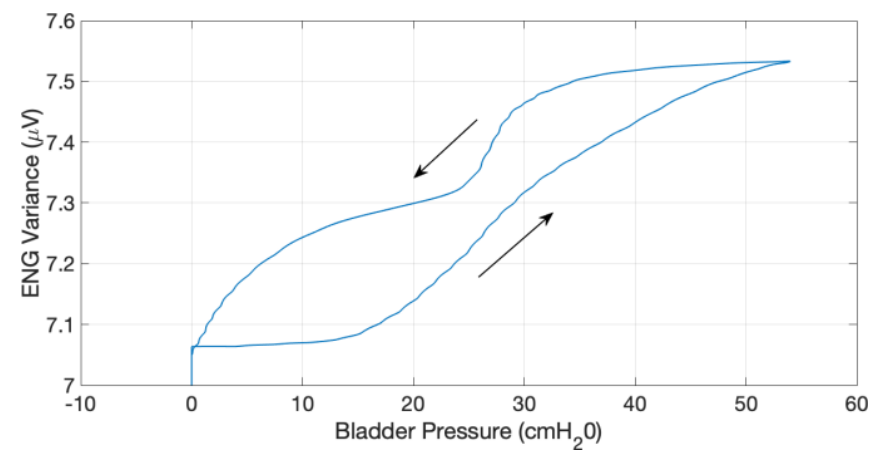

Figure 4: Phase plot of bladder pressure versus ENG variance. Note the hysteresis (arrows indicate direction of travel) and the clear notch when the catheter was opened.

Figure 4 illustrates the relationship between bladder pressure and ENG variance more clearly by using a phase plot. The arrows indicate the direction of travel around the plot and clearly show that there is some hysteresis in the measurement. The sharp vertical trajectory near zero is a result of the ENG variance dropping below the starting value.

\section{DISCUSSION AND CONCLUSIONS}

It appears to be possible to record neural signals that encode bladder pressure from the extradural sacral roots using an implantable cuff electrode. This result is in line with early work performed in pigs, and in both cases the apparent change in ENG variance is incredibly small [6]. The use of delay-andadd as a simple technique to boost the signal amplitude appears to provide some benefit, however the benefit may be limited by the electrode spacing made possible in such a small surgical space. At present there is little information available about the optimal electrode geometry for recording specific velocities that might help inform the design of the cuffs.
The phase plot of Fig. 4 clearly shows hysteresis, which is also in agreement with existing literature [6]. This is perhaps not surprising as the tissues of the bladder are unlikely to contract as rapidly as they stretch during filling, and this behaviour is in fact quite common even in artificial pressure sensors. There are many signal processing methods available for dealing with this situation - such as a generalised PrandtlIshlinskii model [9].

Future work will involve repeating the experimental method described in this paper on a larger number of animals, and examining if the recording is repeatable from one individual to another. Rudimentary validation has been performed by cutting the root proximal to the bladder, but it will be necessary also to record from multiple roots and identify that the correlation with bladder pressure only occurs in those roots that ultimately innervate the bladder.

It remains to be seen if whole-nerve recordings from the sacral roots can detect reflex voiding, and if the same recording can be made in a freely moving animal. Such chronic recordings in animals recovered after the surgical MEC implant described here are currently in progress. This paper has shown that it is possible to make a measurement of urinary bladder activity in sheep using an implantable electrode, and that the use of velocity selective recording can significantly enhance this measurement.

\section{REFERENCES}

[1] M. Fehlings, A. Singh, L. Tetreault, S. Kalsi-Ryan, and A. Nouri, "Global prevalence and incidence of traumatic spinal cord injury," Clin. Epidemiol., p. 309, Sep. 2014.

[2] C.-W. Liu, K. H. Attar, A. Gall, J. Shah, and M. Craggs, "The relationship between bladder management and health-related quality of life in patients with spinal cord injury in the UK," Spinal Cord, vol. 48, no. 4, pp. 319-324, Apr. 2010.

[3] B. Schurch, C. Tawadros, and S. Carda, "Dysfunction of lower urinary tract in patients with spinal cord injury," 2015, pp. $247-$ 267.

[4] J.-J. Wyndaele, A. Brauner, S. E. Geerlings, K. Bela, T. Peter, and T. E. Bjerklund-Johanson, "Clean intermittent catheterization and urinary tract infection: review and guide for future research," BJU Int., vol. 110, no. 11c, pp. E910-E917, Dec. 2012.

[5] G. S. Brindley, "The first 500 patients with sacral anterior root stimulator implants: general description," Spinal Cord, vol. 32, no. 12, pp. 795-805, Dec. 1994.

[6] S. Jezernick, J. Wen, N. Rijkhoff, J. Djurhuusd, and T. Sinkjaer, "Analysis of Bladder Related Nerve Cuff Electrode Recordings from Preganglionic Pelvic Nerve and Sacral Roots in Pigs," $J$. Urol., vol. 163, no. 4, pp. 1309-1314, Apr. 2000.

[7] N. Donaldson, R. Rieger, M. Schuettler, and J. Taylor, "Noise and selectivity of velocity-selective multi-electrode nerve cuffs.," Med. Biol. Eng. Comput., vol. 46, no. 10, pp. 1005-18, Oct. 2008.

[8] G. Schalow, G. Zäch, and R. Warzok, "Classification of human peripheral nerve fibre groups by conduction velocity and nerve fibre diameter is preserved following spinal cord lesion," J. Auton. Nerv. Syst., vol. 1838, no. 6, 1995.

[9] J. Gan, X. Zhang, and H. Wu, “A generalized Prandtl-Ishlinskii model for characterizing the rate-independent and rate-dependent hysteresis of piezoelectric actuators," Rev. Sci. Instrum., vol. 87, no. 3, p. 035002 , Mar. 2016. 